\title{
Interference Suppression and Resource Allocation Strategies Based on IoT Monitoring
}

\author{
Bin $\mathrm{Wu}^{1}$, Bingxin $\mathrm{Yao}^{2 *}$, Yin Yang ${ }^{1}$, Chaoran Zhou ${ }^{1}$, Ning Zhu ${ }^{1}$ \\ ${ }^{1}$ Nanjing Inrich Technology Co., Ltd., Nanjing 210018, China \\ ${ }^{2}$ School of computer science and technology, Nanjing Tech University, Nanjing 211816, China \\ *Email: bingxinyyy@163.com
}

Received: March 9, 2021. Revised: August 1, 2021. Accepted: August 19, 2021. Published: August 24, 2021.

\begin{abstract}
The present application scenarios of the Internet of Things (IoT) often require the equipment to be adaptable, the resource allocation to be efficient, and the signal monitoring and transmission to be effective. However, the existing algorithms cannot solve the problem of system capacity reduction caused by the mutual interference between regions in data rates. Aiming at effectively improving the performance of the IoT monitoring system and ensuring the fairness of each monitoring terminal, this paper attempts to explore interference suppression and resource allocation strategies based on IoT monitoring. First, the paper established an IoT monitoring network model, and elaborated on interference suppression strategies for inter-layer interferences of "Macro Base Station (BS) - Micro Cells" and "Micro BS - Macro Cells" and for intra-layer interference that include the interference between local monitoring networks and interference between terminals in local area networks; then, the paper proposed a sub-carrier resource allocation scheme for IoT monitoring system with multiple inputs and outputs and a waterfilling strategy of system channel power; at last, experimental results verified the effectiveness of the proposed interference suppression and resource allocation algorithm.
\end{abstract}

Keywords-Internet of Things (IoT) monitoring; interference suppression; resource allocation

\section{INTRODUCTION}

A ccording to the prediction reports of Ericsson and Cisco, global networked devices will exceed 600 billion after 2035 , and what's accompanying such rapid increase is the growing IoT resource cost and energy consumption, which has already become an urgent problem to be solved for field scholars [1-3]. For IoT monitoring systems in which the various IoT components present a scalable and highly free distribution state in the small and medium-sized monitoring area, technically, their application scenarios would require the equipment to be adaptable, the resource allocation to be efficient, and the signal monitoring and transmission to be effective [4-6]. Therefore, facing the challenges brought by the rapid growth of data transmission rate and quality of IoT [7-10], it's quite necessary to research the interference suppression and resource allocation strategies in IoT monitoring scenarios.

Current researches concerning interference suppression and resource allocation strategies have already obtained a few results. As one of the key technologies of $5 \mathrm{G}$, the Small Cell network has the advantages of high capacity, wide coverage, small cost, and low energy consumption; however, there're still a few unsolved issues with it, such as system capacity reduction and poor user experience cause by resource allocation and interference problems [11-15]. In order to effectively increase the system capacity of Small Cell network, Yu et al. [16] built a networked hierarchical structure based on users' competition and cooperation behaviors when using wireless resources, they employed an improved particle swarm optimization (PSO) algorithm to adjust the information transmission efficiency of the network, and proposed a sub-channel allocation algorithm based on information transmission power allocation. According to the principle of Overlapping Coalition Formation (OCF) in game theory, Cooper et al. [17] constructed a multi-objective optimization model of Small Cell network sub-channel resource allocation with total data rate and total transmission power as the optimization objectives, and proposed a hierarchical cluster Small Cell BS cooperation scheme for different application scenarios with sparse or dense deployment. Multi-BS cooperation can effectively suppress the interference of users at the edge of the coverage area of BS. Ahmet Ihsan Canbolat and Kazuhiko Fukawa determined the beam downtilt and communication throughput of the far BS and the near BS using genetic algorithm (GA), and built a radiation-propagation model of active antenna array based on the calculation results, the beam-based power allocation strategy they proposed has greatly increased the capacity of the communication system. To meet the business requirements of IoT communication, now the large-scale multi-input/multi-output technology has attracted more attention of global scholars [18-21]. Ding and Liu [22] constructed a two-layer heterogeneous network combining the frequency division duplex (FDD) long-term evolution (LTE) 
and time division duplex (TDD) LTE, by deploying low-power nodes in hot spot and blind spot areas, they have achieved flexible allocation of spectrum resources and hot spot and blind spot compensation of BS deployment. Yang et al. [23] used the Lagrangian multiplier algorithm to allocate the optimal resource block for each user and calculated the specific power value, this research realized effective control of downlink power among users, and gave the corresponding interference suppression scheme. Boulos et al. [24] studied the joint optimization of BS clustering and beamforming in multi-cell and multi-user scenarios, after optimization, they improved the system throughput while effectively suppressing the interference. Peto et al. [25] researched the system channel capacity estimation and resource allocation strategy of Small Cell network in the presence of channel estimation errors, the proposed new strategy has a higher transmission rate and a lower transmission rate for each secondary user. Xiong et al. [26] comprehensively considered the interference threshold of the primary user and the transmission rate and transmission power limitations of secondary users, and proposed a resource allocation algorithm that suppresses the interference of secondary users to the primary users while ensuring its proportional rate.

With the increase in the volume and dimensions of IoT monitoring network resources, the complexity of network interference suppression and resource allocation algorithms increases greatly as well. The existing algorithms cannot solve the problem of system capacity reduction caused by the mutual interference between regions in data rates. To effectively improve the performance of the IoT monitoring system and ensure the fairness of each monitoring terminal, this paper proposed new strategies for interference suppression and resource allocation. The main content of the paper includes: (1) construction of the IoT monitory network model and classification of interference existing in the system; (2) explanation of interference suppression strategies for interlayer interference of "Macro BS - Micro Cells" and "Micro BS - Macro Cells" and for intra-layer interference that include the interference between local monitoring networks and interference between terminals in local area networks; (3) proposal of the sub-carrier resource allocation scheme for IoT monitoring system with multiple inputs and outputs; (4) proposal of the water-filling strategy of system channel power. At last, experimental results verified the effectiveness of the proposed interference suppression and resource allocation algorithm.

\section{INTERFERENCE SUPPRESSION OF IOT MONITORING SYSTEM}

This section takes the IoT monitoring network as the subject. The IoT monitoring network is a downlink heterogeneous wireless network, which composed of one IoT central macroBS and $S$ IoT monitoring micro-BSs. Figure 1 shows the antenna distribution and configuration of the IoT monitoring system model. The macro-BS and each micro-BS are respectively equipped with $n$ and $m$ large-scale antennas, and simultaneously serve $L_{N}$ macro terminals and up to $m$ singleantenna micro terminals. Assume $V_{r}$ represents the number of all micro terminals, the $i$-th micro-BS serves a total of $L_{r i}$ terminals. In order to improve the per unit area spectrum utilization of the IoT monitoring network, it's assumed that the local monitoring network shares the spectrum resources. At this time, there are two types of interference in the system, the interlayer interference of "Macro BS - Micro Cells" and "Micro BS - Macro Cells", and the intra-layer interference that include the interference between local monitoring networks and the interference between terminals in local area networks;

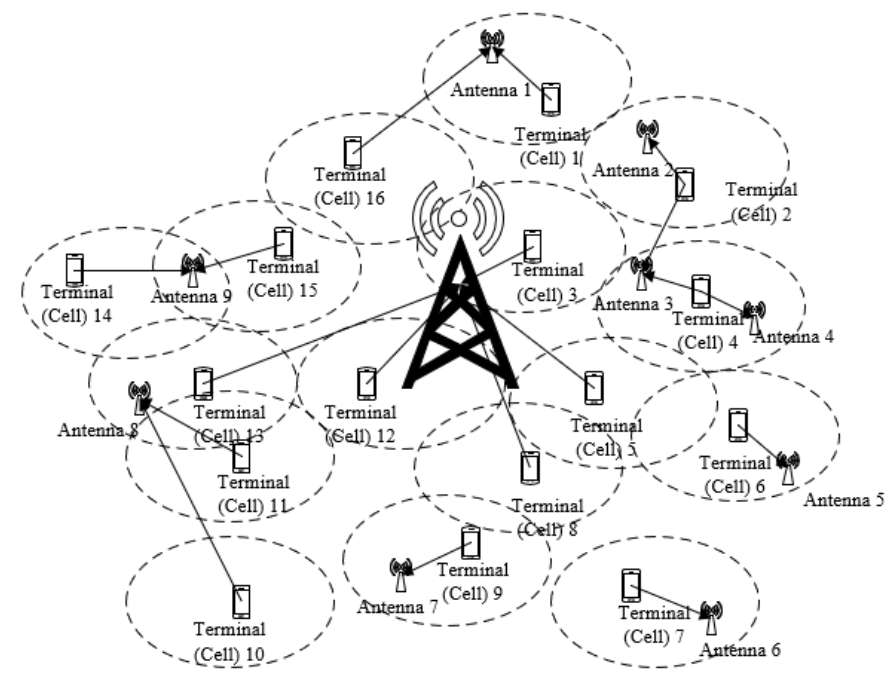

Fig. 1 A diagram of antenna distribution and configuration of the IoT monitoring system model

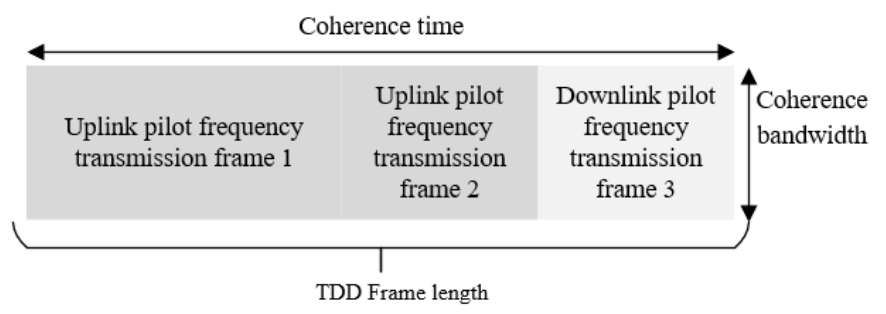

Fig. 2 TDD frame structure

The interference suppression effect of the IoT monitoring system is greatly affected by the accuracy of the acquired channel state. Instead of the traditional FDD form, this study chose to implement the more convenient TDD form. Figure 2 shows the TDD frame structure. Assume: in the IoT monitoring network, the channel state of the macro BS and the micro BSs is ideal; $W_{N}$ and $W_{r M}$ respectively represent the transmitting power of the macro BS and the transmitting power of the $M$-th micro BS; $\delta_{O N l}$ and $D C_{0 N l} \in O^{1 \times n}$ respectively represent the distance between the macro terminal $l$ and the macro BS and the downlink channel matrix; $\delta_{M N l}$ and $D C_{M N l} \in O^{1 \times m}$ respectively represent the distance between the macro terminal $l$ and the $M$ th micro BS and the downlink channel matrix; $\beta$ represents the path loss coefficient, its value is greater than 2; $P R_{0 l} \in O^{n \times 1}$ represents the precoding vector of the macro terminal $1, P R_{M k} \in$ $O^{m \times 1}$ represents the precoding vector of the $k$-th micro terminal served by the $M$-th micro BS; $a_{0 l}$ represents the signal transmitted by the macro BS to macro terminal $1, a_{M k}$ represents 
the signal transmitted by the $M$-th micro BS to the $k$-th micro BS; then, under the condition of equally distributed power, the received signal of the macro terminal $l$ of the IoT monitoring network can be expressed as:

$$
\begin{aligned}
& b_{N l}=\sqrt{\frac{W_{N}}{L_{N}} \delta_{0 N l}^{-\beta}} D C_{0 N l} P R_{0 l} a_{0 l} \\
& +\sum_{j \neq l}^{L_{N}} \sqrt{\frac{W_{N}}{L_{N}} \delta_{0 N l}^{-\beta}} D C_{0 N l} P R_{0 j} a_{0 j} \\
& +\sum_{M=1}^{R} \sum_{k=1}^{L_{r N}} \sqrt{\frac{W_{r M}}{L_{r M}} \delta_{M N l}^{-\beta}} D C_{M N l} P R_{M k} a_{M k}
\end{aligned}
$$

$\delta_{0 r l}$ and $D C_{0 r l} \in O^{1 \times n}$ respectively represent the distance between the micro terminal $l$ and the macro BS and the downlink channel matrix; $\delta_{i r l}$ and $D C_{i r l} \in O^{1 \times m}$ respectively represent the distance between the micro terminal $l$ and the $M$ th micro BS and the downlink channel matrix, then, under the condition of equally distributed power, the received signal of the micro terminal $l$ of the IoT monitoring network can be expressed as:

$$
\begin{aligned}
& b_{r l}=\sum_{i \in O_{l}} \sqrt{\frac{W_{r i}}{L_{r i}} \delta_{i r l}^{-\beta}} D C_{i r l} P R_{i l} a_{r l} \\
& +\sum_{i \in O_{l}} \sum_{v \neq l}^{L_{r i}} \sqrt{\frac{W_{r i}}{L_{r i}} \delta_{i r l}^{-\beta}} D C_{i r l} P R_{i v} a_{i v} \\
& +\sum_{e \notin O_{l}} \sum_{k=1}^{L_{r e}} \sqrt{\frac{W_{r e}}{L_{r e}} \delta_{e r l}^{-\beta}} D C_{e r l} P R_{e k} a_{e k} \\
& +\sum_{c=1}^{L_{N}} \sqrt{\frac{W_{N}}{L_{N}} \delta_{0 r k}^{-\beta}} D C_{0 r l} P R_{0 c} a_{0 c}+M_{r l}
\end{aligned}
$$

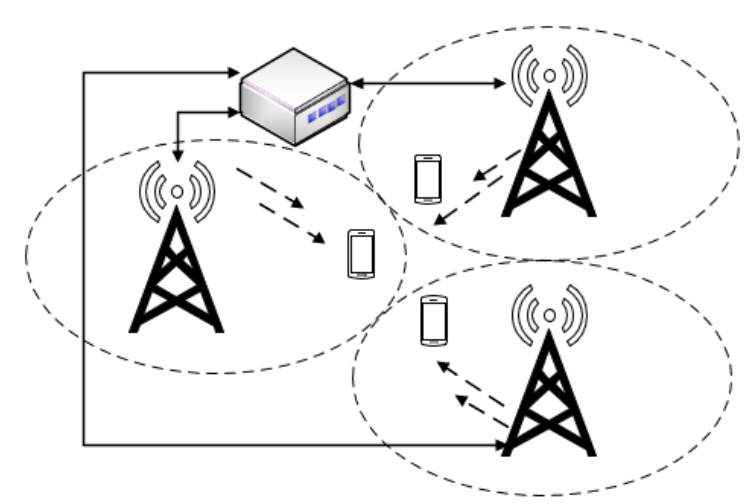

Fig. 3 Local network interference suppression

Figure 3 gives a schematic diagram of local network interference suppression. For intra-layer interference including the interference between local monitoring networks and the interference between terminals in local networks, this paper adopted an interference suppression method based on precoding design. The constraints that the downlink channel matrix and precoding vector of the macro terminal $l$ and the macro BS must meet include:

$$
D C_{0 N l} P R_{0 j}=0, \forall l \neq j, j=\left\{1,2, \ldots, L_{N}\right\},
$$

The constraints that the downlink channel matrix and precoding vector of the micro terminal $l$ and the $M$-th micro BS must meet include:

$$
D C_{i r l} P R_{i v}=0, \forall l \neq v, i=\{1,2, \ldots, R\}, v=\left\{1,2, \ldots, L_{r i}\right\},
$$

The constraints that the downlink channel matrix and precoding vector of the micro terminal $l$ and the macro BS must meet include:

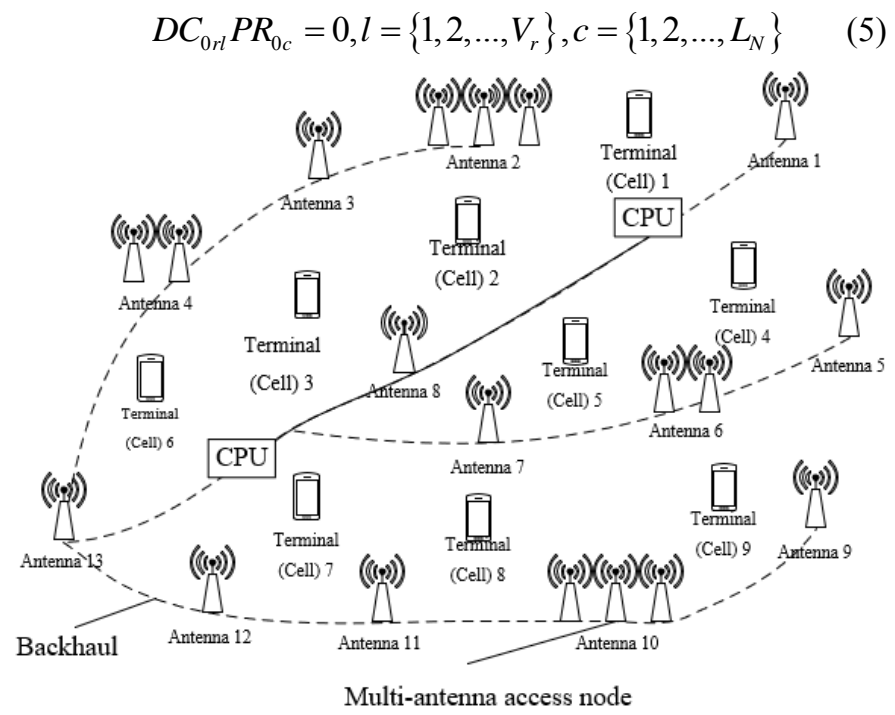

Fig. 4 Joint multi-domain large-scale IoT monitoring system model

Figure 4 gives the model of joint multi-domain large-scale IoT monitoring system. For such system, in order to eliminate the interference of system macro BS to all micro terminals, it's assumed that the system macro BS that was subject to precoding design has more degrees of freedom, $P R^{W}{ }_{0 l}$ represents the precoding vector of the macro terminal $l$ that satisfies formulas 3 and $4 ; M_{N l}$ represents the additive white Gaussian noise and $s^{2}$ represents the variance, then Formula 6 shows the calculation formulas for the data transmission rate of the macro and micro terminals:

$$
\begin{aligned}
& D A_{N l}^{W}= \\
& \log _{2}\left(\begin{array}{l}
\frac{W_{N}}{L_{N}} \delta_{0 N l}^{-\beta} D C_{0 N l} P R_{0 l}^{W}\left(D C_{0 N l} P R_{0 l}^{W}\right)^{H} \\
\sum_{M=1}^{R} \sum_{k=1}^{L_{r M}} \frac{W_{r M}}{L_{r M}} \delta_{M N l}^{-\beta} D C_{M N l} P R_{M k} \\
\left.D C_{M N l}^{W} P R_{M k}\right)^{H}+s^{2}
\end{array}\right) \\
& \log _{2}\left(\begin{array}{c}
\frac{1+}{\sum_{i \in O_{l}} \frac{W_{r i}}{L_{r i}} \delta_{i r l}^{-\beta} D C_{i r l} P R_{i l}\left(D C_{i r l} P R_{i l}\right)^{H}} \\
\sum_{e \notin O_{l}} \sum_{k=1}^{L_{r e}} \frac{W_{r e}}{L_{r e}} \delta_{e r l}^{-\beta} D C_{e r l} P R_{e k} \\
\left(D C_{e r l} P R_{e k}\right)^{H}+s^{2}
\end{array}\right)
\end{aligned}
$$

If the traditional almost blank subframe technology is adopted to eliminate the inter-layer interference of "macro BS micro terminals" and "micro BS - macro terminals", the 
transmitting power of the macro $\mathrm{BS}$ in the IoT monitoring network is 0 , that is, $W_{N}$ is equal to 0 . In such case, the corresponding precoding design only needs to satisfy constraints 3 and 5 to realize the suppression of interference between terminals in local networks. Assume $P R^{m u}{ }_{0 l}$ represents the precoding of the macro terminal 1 that satisfies constraint 3 , $\eta_{W}$ represents the scale coefficient of the almost blank subframe technology after normalization and its value range is $[0,1]$, then Formulas 7 and 8 give the corresponding macro terminal and micro terminal data transmission rate calculation formulas:

$$
\begin{aligned}
& D A_{N l}^{m u}= \\
& \left(1-\eta_{W}\right) \log _{2}\left(\begin{array}{l}
1 \\
\sum_{M=1}^{R} \sum_{k=1}^{L_{r M}} \frac{W_{r M}}{L_{r M}} \delta_{M N l}^{-\beta} D C_{M N l} P R_{M k} \\
\left(D C_{M N l} P R_{M k}\right)^{G}+s^{2}
\end{array}\right) \\
& D A_{r l}^{m u}=\eta_{W} R_{N l}^{W}+\left(1-\eta_{W}\right) \log _{2} \\
& \left(\begin{array}{c}
1+\frac{\sum_{i \in O_{l}} \frac{W_{r i}}{L_{r i}} \delta_{i r l}^{-\beta} D C_{i r l} P R_{i l}\left(D C_{i r l} P R_{i l}\right)^{G}}{\sum_{e \notin O_{l}} \sum_{k=1}^{L_{r e}} \frac{W_{r e}}{L_{r e}} \delta_{e r l}^{-\beta} D C_{e r l} P R_{e k}\left(D C_{e r l} P R_{e k}\right)^{G}} \\
+\sum_{c=1}^{L_{N}} \frac{W_{N}}{L_{N}} \delta_{0 N l}^{-\beta} D C_{0 N l} P R_{0 l}^{W}\left(D C_{0 N l} P R_{0 l}^{W}\right)^{G}
\end{array}\right)
\end{aligned}
$$

After applying the above two types of interference suppression strategies to the IoT monitoring network, in order to maximize the data transmission rate of the network, this paper adopted the power adjustment strategy to suppress the two types of interference, namely the fewer interference between local monitoring networks, and the interference of "micro BS - macro terminals". Assume $W_{r}=\left(w_{r k}, w_{r 2}, \ldots, w_{r R}\right)^{T}$ represents the collection of the data transmission power of each micro BS, wherein $w_{r}^{\max }$ represents the maximum data transmission power, then Formula 9 gives the optimized interference suppression model of joint multi-domain largescale IoT monitoring system:

$$
\begin{aligned}
& \max _{w_{r}, \eta_{W}, \omega_{1}, \omega_{2}} \omega_{1}\left(\sum_{l=1}^{L_{N}} D A_{N l}^{W}+\sum_{l=1}^{V_{r}} D A_{r l}^{W}\right) \\
& +\omega_{2}\left(\sum_{l=1}^{L_{N}} D A_{N l}^{m u}+\sum_{l=1}^{V_{r}} D A_{r l}^{m u}\right)
\end{aligned}
$$

The constraints that the precoding vector of macro terminal 1 that satisfies Formulas 3 and 4 and the downlink channel matrix of macro terminal 1 and macro BS must meet include:

$$
\text { s.t. } D C_{0 N l} P R_{0 j}^{W}=0, \forall l \neq j, j=\left\{1,2, \ldots, L_{N}\right\}
$$

The constraints that the precoding vector of micro terminal 1 and the downlink channel matrix of micro terminal 1 and the $M$-th micro BS must meet include:

$$
D C_{i r l} P R_{i v}=0, \forall l \neq v, i=\{1,2, \ldots, R\}, v=\left\{1,2, \ldots, L_{r i}\right\}
$$

The constraints that the downlink channel matrix of micro terminal 1 and macro BS and the precoding vector of macro terminal 1 that satisfies formulas 3 and 4 must meet include:

$$
D C_{0 r l} P R_{0 c}^{W}=0, l=\left\{1,2, \ldots, V_{r}\right\}, c=\left\{1,2, \ldots, L_{N}\right\}
$$

The constraints that the downlink channel matrix of macro terminal 1 and macro BS and the precoding vector of macro terminal 1 that satisfies Formula 3 must meet include:

$$
D C_{0 N l} P R_{0 j}^{m u}=0, \forall l \neq j, j=\left\{1,2, \ldots, L_{N}\right\}
$$

Formulas 14 and 15 show the conditions for the selection principles of the two types of inter-layer interference management methods that constrain the almost blank sub-frame technology and the code domain:

$$
\begin{aligned}
& \omega_{1}, \omega_{2} \in\{0,1\} \\
& \omega_{1}+\omega_{2} \leq 1
\end{aligned}
$$

Formula 16 is the condition that the scale coefficient $\eta_{W}$ needs to meet:

$$
0 \leq \eta_{W} \leq 1
$$

The data transmission power of each micro BS needs to meet:

$$
0 \leq W_{r k}(k=1,2, \ldots, R) \leq W_{r}^{\max }
$$

\section{RESOURCE ALLOCATION STRATEGIES FOR THE IOT MONITORING SYSTEM}

In an IoT monitoring system with multiple inputs and outputs, reasonably allocating the limited spectrum resources for dense monitoring areas is a very important task. With the increase in the number of required monitoring areas, in order to effectively improve the utilization of power resources, it is necessary to effectively and fully utilize and save the power resources, and this is of great significance to the application of the resource-constrained IoT monitoring scenarios. The current resource allocation strategies are mainly divided into two types, one is system capacity maximization, and the other is transmitting power minimization. The former is mainly achieved by maximizing the signal-to-noise ratio, and the latter is mainly achieved by controlling the data volume carried by each sub-carrier and the state of the channels. This paper mainly researched the sub-carrier resource allocation technology based on IoT monitoring system capacity maximization, and Figure 5 gives the workflow of resource allocation of the IoT monitoring system.

Figure 6 shows the distribution and configuration of $\mathrm{BS}$ antennas. Assume there're $m_{F S}$ and $m_{J S}$ transmitting antennas and receiving antennas in the $\mathrm{BS}$ of the IoT monitoring system, $W_{A C}$ represents the total power of information transmission, $L$ represents the number of monitored terminals, $M_{S}$ represents the number of sub-carriers, $T W$ represents the bandwidth; $G_{l, m S}$ represents the $m_{J S} \times m_{F S}$ dimensional frequency response matrix of terminal 1 on sub-carrier $m_{S} ; a_{l, m S}$ and $b_{l, m S}$ represent the corresponding $m_{F S} \times 1$ dimensional sub-carrier transmitted signal vector and the $m_{J S} \times 1$ dimensional sub-carrier received signal vector; $G N_{l, m S}$ represents the $m_{J S} \times 1$ dimensional cyclic symmetric Gaussian noise; $m_{0}$ represents the power spectrum density of the additive white Gaussian noise, if the terminal changes, the channel response of the system changes 
accordingly, and the signal received by terminal 1 can be expressed by Formula 18:

$$
b_{l, m_{s}}=G_{l, m_{s}} a_{l, m_{s}}+G N_{l, m_{s}}, m_{s}=1,2, \ldots, M_{s}
$$

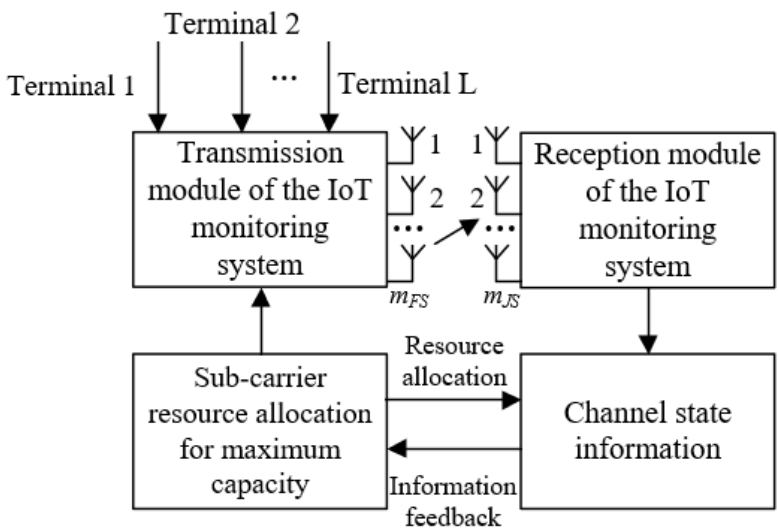

Fig. 5 Workflow of resource allocation of the IoT monitoring system

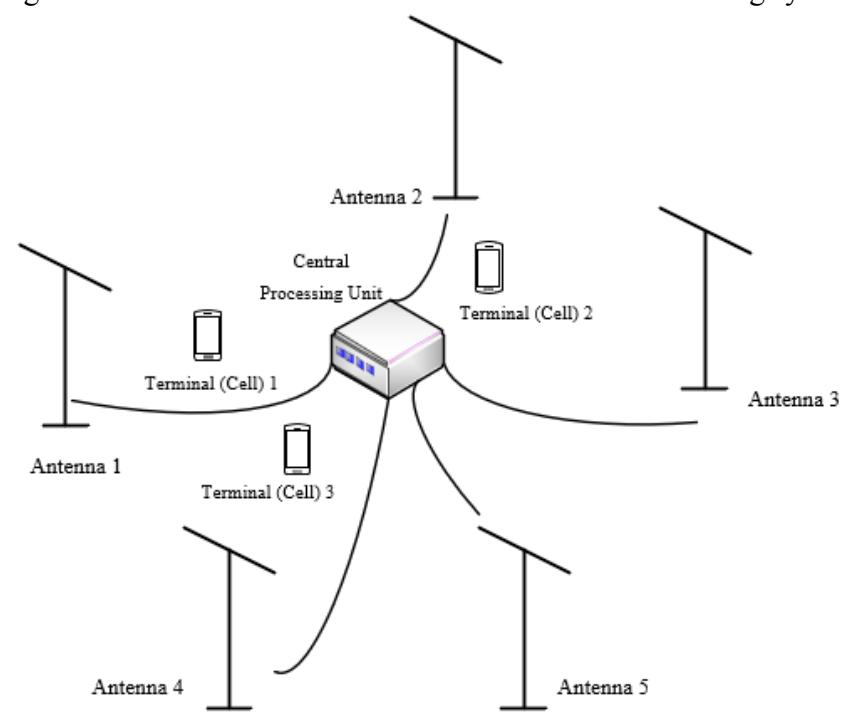

Fig. 6 Distribution and configuration of BS antennas

When the sub-carriers are allocated to the terminals, the IoT monitoring system at this time can be regarded as a multi-input system with a single monitoring terminal, and the multi-input and multi-output system capacity of multiple monitoring terminals can be equivalent to the sum of the system capacity of single monitoring terminals. Assume $G_{l, m s}$ and $W_{l, m s}$ represent the channel gain matrix and the transmitting power of terminal $l$ on subcarrier $m_{S}$, and $R=\min \left(m_{J S}, m_{F S}\right)$ represents the rank of $G_{l, m S}$, then by performing singular value decomposition on $G_{l, m S} G_{l, m S}^{H}$, the eigenvalue $\left\{\mu_{l}^{i}, m_{S}\right\}^{n E_{i=1}}$ can be obtained. Whether to allocate sub-carrier $m_{S}$ to terminal $l$ is represented by a binary function $\rho_{l, m S}$, if yes, it takes 1 , otherwise it takes 0 ; then, the instantaneous capacity of the terminals of the IoT monitoring system can be calculated by Formula 18:

$$
\begin{aligned}
& M O= \\
& \sum_{l=1}^{L} \sum_{m_{S}=1}^{M_{S}} \frac{\rho_{l, m_{S}}}{M_{S}} \log _{2}\left[\operatorname{det}\left(I_{m_{J S}}+\frac{W_{l, m_{S}} G_{l, m_{S}} G_{l, m_{S}}^{H}}{T W m_{0} / M_{S}}\right)\right] \\
& =\sum_{l=1}^{L} \sum_{m_{S}=1}^{M_{S}} \sum_{i=1}^{n_{E}} \frac{\rho_{l, m_{S}}}{M_{S}} \log _{2}\left(1+\frac{W_{l, m_{S}} \mu_{l, m_{S}}^{i}}{T W m_{0} / M_{S}}\right)
\end{aligned}
$$

According to the definition principle of Jensen's inequality for convex function, $M O$ needs to satisfy the inequality shown in Formula 20:

$$
\begin{aligned}
& \sum_{l=1}^{L} \sum_{m_{S}=1}^{M_{S}} \sum_{i=1}^{n_{E}} \frac{\rho_{l, m_{S}}}{M_{S}} \log _{2}\left(1+\frac{W_{l, m_{S}} \mu_{l, m_{S}}^{i}}{T W m_{0} / M_{S}}\right) \\
& \leq \sum_{l=1}^{L} \sum_{m_{S}=1}^{M_{S}} \frac{\rho_{l, M} n_{E}}{M_{S}} \log _{2}\left(1+\frac{W_{l, m_{S}}}{T W m_{0} / M_{S}} \frac{1}{n_{E}} \sum_{i=1}^{n_{E}} \mu_{l, m_{S}}^{i}\right) \\
& =\sum_{l=1}^{L} \sum_{m_{S}=1}^{M_{S}} \frac{\rho_{l, m_{S}} n_{E}}{M_{S}} \log _{2}\left(1+\frac{W_{l, m_{S}}}{T W m_{0} / M_{S}} \frac{1}{n_{E}} \sum_{i=1}^{n_{E}} \operatorname{tr}\left(G_{l, m_{S}} G_{l, m_{S}}^{H}\right)\right) \\
& =\sum_{l=1}^{L} \sum_{m_{S}=1}^{M_{S}} \frac{\rho_{l, m_{S}} n_{E}}{M_{S}} \log _{2} \\
& \left.1+\frac{W_{l, m_{S}}}{T W m_{0} / M_{S}} \frac{1}{n_{E}}\left\|G_{l, m_{S}}\right\|_{F}^{2}\right)
\end{aligned}
$$

According to above formula, the state of the channel matrix of terminal $l$ on sub-carrier $m_{S}$ can be described by the F-norm of $G_{l, m s}$, then, the subcarrier allocation criterion that meets the requirements can be derived, that is, maximizing the capacity of the IoT monitoring system by allocating $m_{S}$ to the largest terminal 1 of $\left\|G_{l},{ }_{M}\right\|^{2}$. Assume $\Lambda\left(m_{F S} m_{J S}\right)$ represents the $\gamma$ function, and it satisfies $\Lambda\left(m_{F S} m_{J S}\right)=\left(m_{F S} m_{J S}-1\right)$ !; if there is a random variable whose channel matrix norm satisfies the chisquare test distribution and the degree of freedom is $2 m_{F S} m_{J S}$, then its probability density function $h_{F D}(a)$ is:

$$
h_{F D}(a)=\frac{1}{\Lambda\left(m_{F S} m_{J S}\right)} a^{m_{F S} m_{J S}-1} \exp (-a)
$$

The cumulative distribution function (CDF) of this variable can be expressed by Formula 22:

$$
H_{F D}(a)=1-\exp (-a) \sum_{i \in 1}^{m_{F S} m_{I S}-1} \frac{1}{i !} a^{i}
$$

Formula 23 gives the CDF of the instantaneous channel capacity of terminals in the IoT monitoring system on $m_{s}$ :

$$
H_{M O}(b)=\left[H_{F D}\left(\frac{2^{b / n_{E}}-1}{\frac{W_{l, m_{S}}}{m_{0} T W / M_{S}} / n_{E}}\right)\right]^{l}
$$

If it is assumed that the system has a high signal-to-noise ratio, Formula 20 can be simplified as shown in Formula 24 below: 


$$
\begin{aligned}
& \sum_{l=1}^{L} \sum_{m_{S}=1}^{M_{S}} \sum_{i=1}^{n_{E}} \frac{\rho_{l, m_{S}}}{M_{S}} \log _{2}\left(1+\frac{W_{l, m_{S}} \mu_{l, m_{S}}^{i}}{T W m_{0} / M_{S}}\right) \\
& \approx \sum_{l=1}^{L} \sum_{m_{S}=1}^{M_{S}} \frac{\rho_{l, m_{S}}}{M_{S}} \log _{2}\left(\prod_{i}^{n_{E}} \frac{W_{l, m_{S}} \mu_{l, m_{S}}^{i}}{T W m_{0} / M_{S}}\right) \\
& =\log _{2}\left[\left(\frac{W_{l, m_{S}}}{T W m_{0} / M_{S}}\right)^{n_{E}} \operatorname{det}\left(G_{l, m_{S}} G_{l, m_{S}}^{H}\right)\right]
\end{aligned}
$$

According to above formula, $\operatorname{det}\left(G_{l, m S} G^{H} l, m s\right)$ can be used to describe the maximum capacity that can be achieved on each sub-carrier under the above-mentioned conditions.

In a multi-terminal IoT monitoring system, the interference between the terminals can be eliminated based on the ideal signal detection algorithm. Assume $\left[G_{l, m S}\right]_{N}$ represents the $N$-th column of $G_{l, m S}, G_{l, m S, N}$ represents the allocated power of terminal 1 on sub-channel $\left(m_{S}, N\right)$, then, the corresponding rate can be expressed as Formula 25:

$$
D A_{l, m_{S}, N}=\log _{2}\left(1+\frac{W_{l, m_{S}, N}}{T W m_{0} / M_{S}}\left\|\left[G_{l, m_{S}}\right]_{N}\right\|_{F}^{2}\right)
$$

Based on the capacity maximization criterion, sub-channel $\left(m_{S}, N\right)$ is allocated to terminal 1 , this process can be described by Formula 26:

$$
\begin{aligned}
& M O= \\
& \begin{array}{c}
\frac{\rho_{l, m_{S}, N}}{M_{S}} \log _{2} \\
\max _{W_{l, m_{S}, N} \omega_{l, m_{S}, N}} \sum_{l=1}^{L} \sum_{m_{S}=1}^{M_{S}} \sum_{N=1}^{m_{F S}}\left(\begin{array}{l}
1+ \\
\frac{W_{l, m_{S}, N}}{T W m_{0} / M_{S}}\left\|\left[G_{l, m_{S}}\right]_{N}\right\|_{F}^{2}
\end{array}\right)
\end{array}
\end{aligned}
$$

Formula 27 gives the total power constraint to be satisfied by the instantaneous capacity of the terminals in the IoT monitoring system:

$$
\sum_{l=1}^{L} \sum_{m_{S}=1}^{M_{S}} \sum_{N=1}^{m_{F S}} W_{l, m_{S}, N}=W_{A}
$$

Formula 28 gives the constraint of whether to allocate subcarrier $m_{S}$ to terminal 1 :

$$
\sum_{l=1}^{L} \omega_{l, m_{S}, N} \leq 1
$$

The water-filling allocation strategy of channel power has the best effect, and it can perform water-filling allocation in space and frequency domains at the same time on the transmitted signal sub-channel of the multi-terminal IoT monitoring system. If the system has $M_{S} \times m_{F S}$ sub-channels, there're a total of $L \cdot L \cdot \ldots \cdot L=L^{M S \times m F S}$ possible allocation combinations. All the allocation combinations are numbered, and the combination sequence number set is denoted as $K=\left\{1,2, \ldots, K^{M S \times m F S}\right\}$. $T^{(k)}=\left\{T^{(k)}{ }_{1,1}, T^{(k)}{ }_{1,2}, \ldots, T^{(k) M S, m F S}\right\}$ represents the set of terminals selected for each channel under the $k$-th allocation combination, $\lambda$ represents the water level of water-filling allocation of all subchannel power, then the power allocated on $\left(m_{S}, N\right)$ can be calculated by Formula 29:

$$
W_{m_{S}, N}^{(1)}=\max \left(0, \lambda-\frac{m_{0} \frac{T W}{M_{S}}}{\left\|\left[G_{m_{S}}^{R_{m_{S}, N}^{(k)}}\right]_{N}\right\|_{F}^{2}}\right)
$$

$W^{(k)} m_{S, N}$ can be obtained from Formula 30 :

$$
\sum_{m_{S}=1}^{M_{S}} \sum_{N=1}^{m_{F S}} W_{m_{S}, N}^{(l)}=W_{T}
$$

Based on the optimal allocation algorithm, the capacity obtained by the terminals in the IoT monitoring system is:

$$
\begin{aligned}
& M O= \\
& \max _{k \in K} \frac{1}{M_{S}} \sum_{m_{S}=1}^{M_{S}} \sum_{N=1}^{m_{F S}} \log _{2}\left(\frac{W_{m_{S}, N}^{(k)}\left\|\left[G_{M}^{R_{m S, N}^{(k)}}\right]_{N}\right\|_{F}^{2}}{T W m_{0} / M_{S}}\right)
\end{aligned}
$$

\section{EXPERIMENTAL RESULTS AND ANALYSIS}

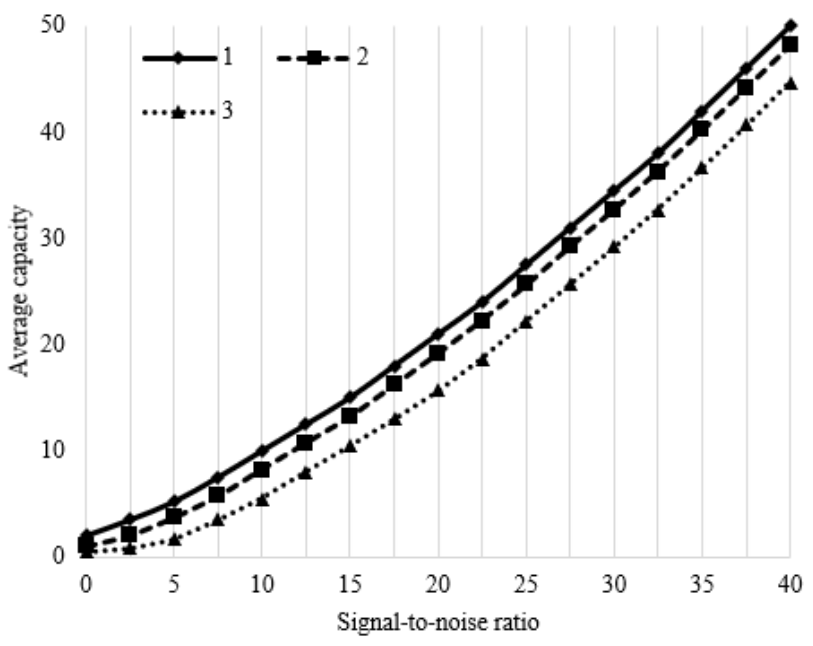

Fig. 7 System capacity under different allocation strategies

This study employed simulation software Matlab to perform simulation experiments on the interference suppression and resource allocation algorithm proposed for the IoT monitoring system. In the experiments, the numbers of transmitting and receiving antennas of the $\mathrm{BS}$ were fixed, and the change laws of the average system capacity under three conditions (maximum sub-carrier F-norm allocation criterion 1, power water-filling allocation criterion 2 , and frequency division multiple address mode allocation criterion 3) were explored, Figure 7 gives the corresponding system capacities. According to the simulation results, the system capacity obtained by the resource allocation algorithm proposed in this paper is higher than that obtained by the traditional static sub-carrier allocation algorithm; and with the increase of the signal-to-noise ratio, the obtained system capacity increases as well. 


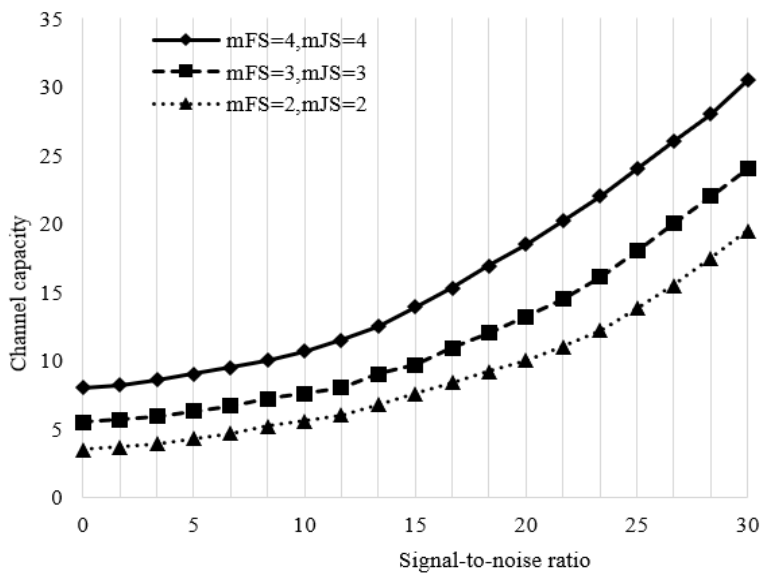

Fig. 8 System capacity under conditions with different antenna numbers

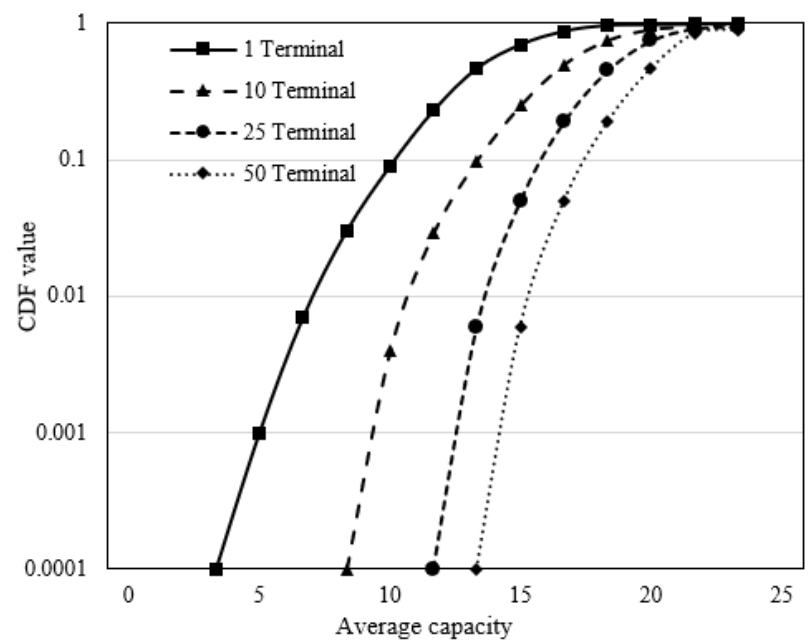

Fig. 9 CDF curves of system capacity under conditions with different terminal numbers

To further explore the relationship between the capacity of the IoT monitoring system and the number of antennas of each BS, comparative simulation experiments were performed and Figure 8 gives the system capacity under conditions with different antenna numbers. According to the figure, the system capacity calculated by the resource allocation algorithm proposed in this paper increases with the number of antennas of each BS, this is because the proposed algorithm provides more degrees of freedom for the multi-antenna system through precoding design, the utilization rate of resources is greatly improved, thus the system channel capacity is increased. Figure 9 shows the CDF curves under conditions with different terminal numbers. According to the figure, the increase of the number of terminals would result in an increase in the multiterminal diversity gain, and the capacity of the IoT monitoring system grows accordingly. At the same time, the CDF curves deduced by Formula 23 basically coincide with the system capacity simulation curves, which can further verify the correctness and accuracy of the theoretical derivation of the algorithm.

In this study, the change law of the average throughput loss of the IoT monitoring system before and after the implementation of macro BS interference suppression strategy was analyzed as well, and Figure 10 shows the curves of the average system throughput loss before and after macro BS interference suppression. According to the figure, since the macro BS has adopted the "Macro BS - Micro cells" interference suppression measures, namely to use the precoding design-based interference suppression method to effectively reduce the inter-beam interference, compared with other cases that didn't adopt interference suppression measures, the proposed algorithm has effectively reduced the average throughput loss of the IoT monitoring system.

Comparative simulation experiments were performed on the IoT monitoring system before and after the implementation of the sub-carrier resource allocation strategy, and Figure 11 gives the average system throughput loss before and after the implementation of the sub-carrier resource allocation strategy. According to the figure, when the average throughput loss of the IoT monitoring system is $6.5 \%$, the adjacent channel interference power ratio required by the subcarrier resource allocation algorithm proposed in this paper is reduced by about $1.2 \mathrm{~dB}$ compared with that without resource allocation, which indicates that the sub-carrier resource allocation has a limited impact on the performance of the IoT monitoring system.

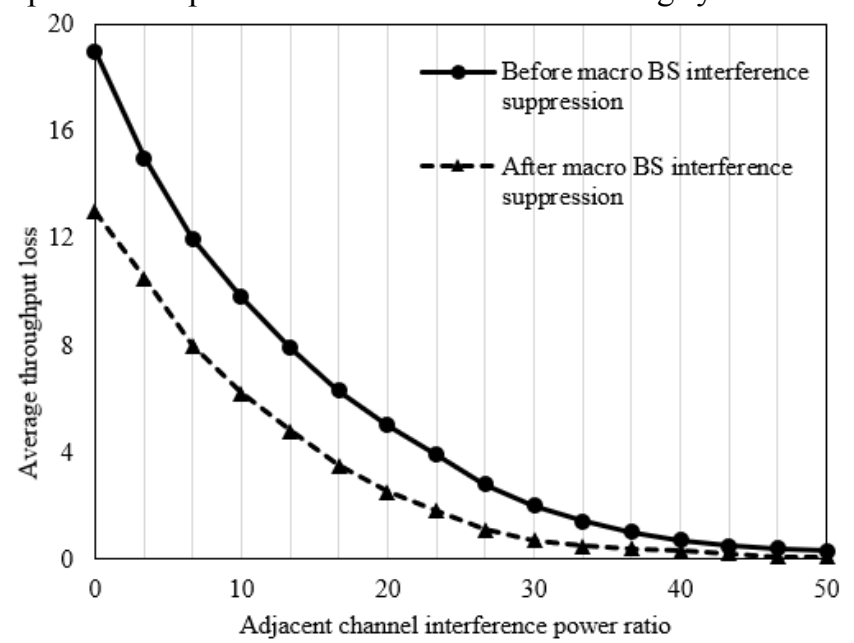

Fig. 10 Average system throughput loss before and after macro BS interference suppression

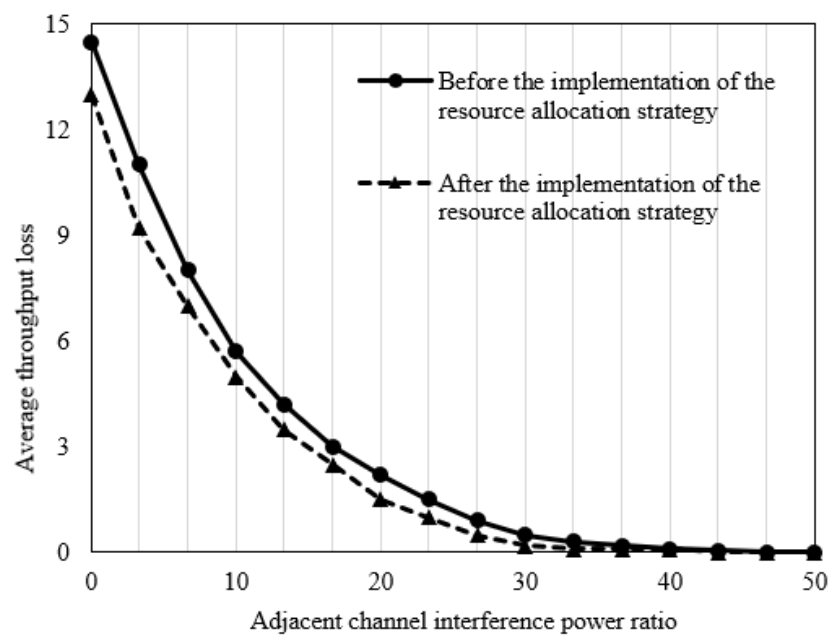

Fig. 11 Average system throughput loss before and after the implementation of resource allocation strategy 
Next, comparative simulation experiments were also performed to verify the effectiveness of the power water-filling allocation criterion, and Figure 12 gives the average system throughput loss under the condition of equal power signal transmission scheme of each BS and under the condition of the proposed algorithm. According to the figure, when there is external interference in the IoT monitoring system, applying power control resource allocation strategy can ensure that the system throughput remains at a relatively high level. When the average system throughput loss is $6 \%$, the adjacent channel interference power ratio required by the proposed algorithm is reduced by about $3.35 \mathrm{~dB}$ compared with that without resource allocation.

Since the interference suppression effect of the IoT monitoring system is greatly affected by the accuracy of the acquired channel state, this study analyzed the influence of spectrum bandwidth conditions on the average system throughput loss under different duplex modes, and Figure 13 gives the average system throughput loss under three conditions: $10 \mathrm{MHz}$ bandwidth FDD-20MHz interference bandwidth TDD system, $10 \mathrm{MHz}$ bandwidth FDD-10MHz interference bandwidth TDD system, and $20 \mathrm{MHz}$ bandwidth FDD- $10 \mathrm{MHz}$ interference bandwidth TDD system. According to the figure, the average throughput loss of systems with a $20 \mathrm{MHz}$ interference bandwidth is greater, and a higher adjacent channel interference power ratio is required to ensure the reduction of inter-system interference. In order to reach $5 \%$ average system throughput loss, the adjacent channel interference power ratio required by the IoT monitoring system with a bandwidth less than $10 \mathrm{MHz}$ is the smallest, about $13.2 \mathrm{~dB}$.

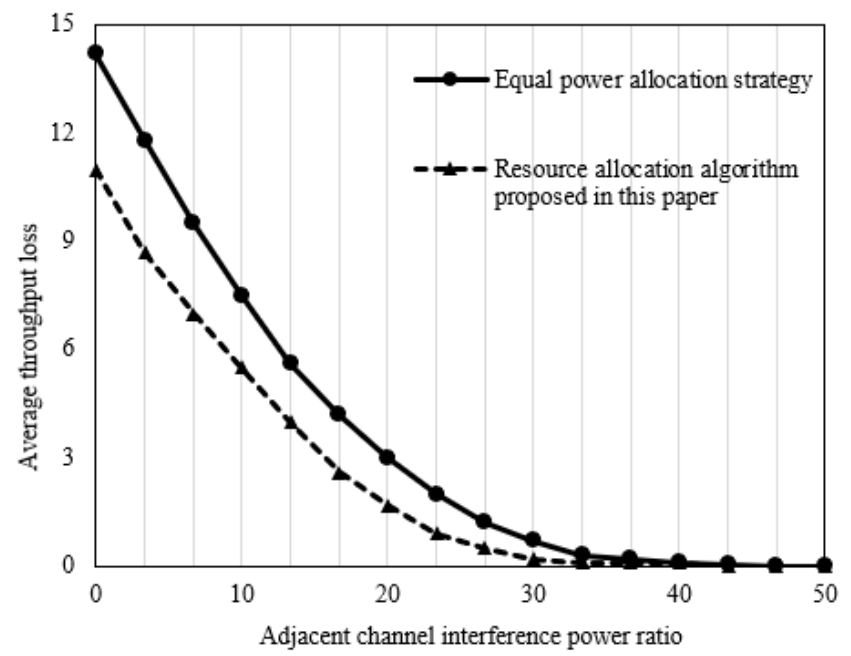

Fig. 12 Average system throughput loss under different resource allocation situations

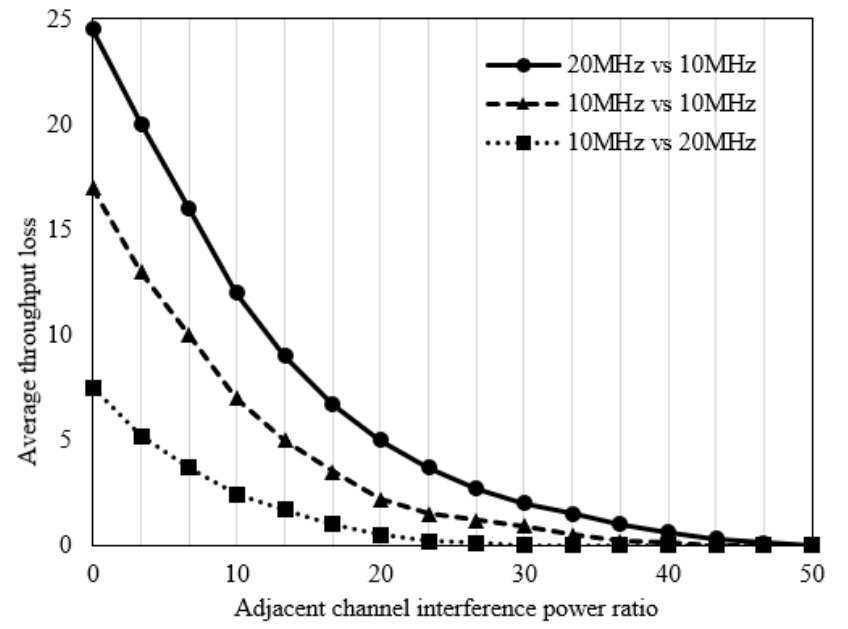

Fig. 13 Average system throughput loss under different bandwidth conditions

\section{CONCLUSION}

This paper researched the interference suppression and resource allocation strategies for IoT monitoring system. First, it established an IoT monitoring network model and elaborated on interference suppression strategies for inter-layer interference and intra-layer interference; then, it proposed a sub-carrier resource allocation scheme for IoT monitoring system with multiple inputs and outputs and a water-filling strategy of system channel power; after that, combining with experiments, the change laws of system capacity under the conditions of different allocation strategies, different antenna numbers, and different terminal numbers were explored, and it's verified that the proposed algorithm can effectively reduce the average throughput loss of the IoT monitoring system. By performing comparative simulation experiments, this paper obtained the average system throughput loss curves before and after macro BS interference suppression, before and after the implementation of resource allocation strategy, and under the conditions of different resource allocation situations and different bandwidth situations; the experimental results proved that the adjacent channel interference power ratio required by the proposed algorithm is lower, and the IoT resource cost and energy consumption reduction effect is better.

\section{References}

[1] M. Winkowski, T. Stacewicz, "Optical interference suppression using wavelength modulation," Optics Communications, vol. 480, pp. 126464, 2021.

[2] L. Zhang, B. Chen, R. Song, W. Song, "Mainlobe interference suppression algorithm based on BMP and L2 norm constraint," In Twelfth International Conference on Signal Processing Systems, International Society for Optics and Photonics, vol. 11719, pp. 117190, 2021.

[3] H. Zhang, M. Liu, "A time-space network based approach for the medical resource order and distribution scheduling problem," ICIC Express Letters, Part B: Applications, vol. 6, no. 7, pp. 1975-1982, 2015.

[4] J. Hu, H. Chen, Z. Lin, H. Li, J. Xie, "Radio frequency interference suppression algorithm based on SOCP in 
OTHR," Circuits, Systems, and Signal Processing, vol. 36, no. 6, pp. 2459-2472, 2017.

[5] M. Chen, S. Yan, S. S. Wang, C. L. Liu, "A generalized network flow model for the multi-mode resourceconstrained project scheduling problem with discounted cash flows," Engineering Optimization, vol. 47, no. 2, pp. $165-183,2015$.

[6] M. J. McGrath, V. Riccobene, G. Petralia, G. Xilouris, M. A. Kourtis, "Performant deployment of a virtualised network functions in a data center environment using resource aware scheduling,” In 2015 IFIP/IEEE International Symposium on Integrated Network Management (IM), pp. 1131-1132, 2015.

[7] M. Clark, K. Psounis, "Efficient resource scheduling for a secondary network in shared spectrum," In 2015 IEEE Conference on Computer Communications (INFOCOM), pp. 1257-1265, 2015.

[8] A. B. Shams, S. R. Abied, M. F. Hossain, "Performance comparison of network layouts with mobile users under different resource scheduling techniques in downlink LTE," In 2016 5th International Conference on Informatics, Electronics and Vision (ICIEV), pp. 949-954, 2016.

[9] T. T. Ran, S. L. Sun, H. Huang, "Cognitive radio based resource scheduling strategy for LTE-A self-organizing network," Lecture Notes of the Institute for Computer Sciences, Social-Informatics and Telecommunications Engineering, LNICST, vol. 149, pp. 26-33, 2015.

[10] T. Sousa, M. A. F. Ghazvini, H. Morais, R. Castro, Z. Vale, "Two-stage stochastic day-ahead optimal resource scheduling in a distribution network with intensive use of distributed energy resources," In 2015 IEEE PES Innovative Smart Grid Technologies Latin America (ISGT LATAM), pp. 689-694, 2015.

[11]N. K. Mangali, P. Venkataram, "Agent Based Call Scheduling Technique with Optimum Resource Utilization in Cellular Controlled Short-Range Communication (CCSRC) Network," In 2015 Fifth International Conference on Communication Systems and Network Technologies, pp. 132-137, 2015.

[12] Y. N. Lien, T. I. Kao, J. S. Huang, "Resource delivery path dependent deployment scheduling for contingency cellular network," In 2014 IEEE International Conference on Pervasive Computing and Communication Workshops (PERCOM WORKSHOPS), pp. 308-313, 2014.

[13] M. Porranzl, C. Wagner, H. Jaeger, A. Stelzer, "HighBandwidth Quasi-Circulator-Based Monostatic Automotive Radar with Self-Interference Suppression," IEEE Transactions on Microwave Theory and Techniques, vol. 69, no. 1, pp. 198-209, 2020.

[14] K. Maruta, C. J. Ahn, "Multi Modulus Signal Adaptation for Semi-Blind Uplink Interference Suppression on Multicell Massive MIMO Systems," IEICE Transactions on Communications, 2020.

[15]E. I. Ackerman, C. H. Cox, "Improved RF Interference Suppression Method," Journal of Lightwave Technology, vol. 38, no. 19, pp. 5546-5550, 2020.

[16] H. Yu, N. Liu, L. Zhang, Q. Li, J. Zhang, S. Tang, S. Zhao, "An interference suppression method for multistatic radar based on noise subspace projection," IEEE Sensors Journal, vol. 20, no. 15, pp. 8797-8805, 2020.

[17] R. J. Cooper, D. W. Prescott, G. J. Lee, K. L. Sauer, "RF atomic magnetometer array with over $40 \mathrm{~dB}$ interference suppression using electron spin resonance," Journal of Magnetic Resonance, vol. 296, pp. 36-46, 2018.

[18] A. I. Canbolat, K. Fukawa, "Joint interference suppression and multiuser detection schemes for multi-cell wireless relay communications: A three-cell case," IEEE Transactions on Communications, vol. 66, no. 4, pp. 13991410, 2017.

[19] S. Lee, J. Y. Lee, S. C. Kim, "Mutual interference suppression using wavelet denoising in automotive FMCW radar systems," IEEE Transactions on Intelligent Transportation Systems, 2019.

[20]K. Shima, K. Maruta, C. J. Ahn, "Data-aided SMI algorithm using common correlation matrix for adaptive array interference suppression," IEICE Transactions on Fundamentals of Electronics, Communications and Computer Sciences, vol. 104, no. 2, pp. 404-411, 2021.

[21] A. Ivanov, A. Osinsky, D. Lakontsev, D. Yarotsky, "High performance interference suppression in multi-user Massive MIMO detector," In 2020 IEEE 91st Vehicular Technology Conference (VTC2020-Spring), pp. 1-5, 2020.

[22]Z. Ding, F. Liu, “A Metamaterial Design for Suppression of Cross Interference in Multi-Frequency Multi-Load MCR WPT System," In 2020 IEEE 9th International Power Electronics and Motion Control Conference (IPEMC2020-ECCE Asia), pp. 90-95, 2020.

[23] S. Yang, Z. Yang, J. Zhang, Q. Zhou, "A Subarray Level Adaptive Interference Suppression Method Based on Particle Swarm Optimization and Greedy Algorithm," In 2020 IEEE 6th International Conference on Computer and Communications (ICCC), 1124-1129, 2020.

[24]F. Boulos, L. Dall'Asta, G. Gottardi, M. A. Hannan, A. Polo, A. Salas-Sanchez, M. Salucci, "A computational inversion method for interference suppression in reconfigurable thinned ring arrays," In Journal of Physics: Conference Series, vol. 1476, no. 1, pp. 12016, 2016.

[25]T. Peto, L. Dudas, R. Seller, "Analog direct path interference suppression for FM based passive radars," In 2018 28th International Conference Radioelektronika (RADIOELEKTRONIKA), pp. 1-6, 2018.

[26] W. Xiong, M. Greco, F. Gini, G. Zhang, Z. Peng, "SFMM design in colocated CS-MIMO radar for jamming and interference joint suppression," IET Radar, Sonar \& Navigation, vol. 12, no. 7, pp. 702-710, 2018.

Bin Wu. He was born in Changzhou City, Jiangsu Province in 1974. He graduated from signal and information processing major of Southeast University and obtained a doctorate in engineering in April 2015. From 2005 to now, he has participated in a number of key $\mathrm{R} \& \mathrm{D}$ projects in the communication and power industries and provincial and municipal scientific research projects.

Bingxin Yao. He was born in 1980. He received the B.S., M.S, and Ph.D. degree in information science and engineering from Southeast University, Nanjing, China in 2002, 2005 and 2009 respectively. From 2010 to now, he was a assistant professor in 
the Department of Computer Science and Technology, Nanjing technology university, working on various problems in signal processing.

Yin Yang. He was born in 1986 in Gaochun County, Jiangsu Province. He Graduated from Nanjing Vocational College of Information Technology in 2007. After graduation, he worked in RF debugging for 5 years, and then in hardware design. Now he is doing the hardware development of the Internet of things system project.

Chaoran Zhou. He was born in Laian County, Chuzhou City, Anhui Province. He graduated from school of electrical and information engineering of Anhui University of science and technology in 2015 .He began to focus on embedded software engineer work in 2015, and now from the Internet of things system development project.

Ning Zhu. She was born in 1994 in Nanjing city, Jiangsu province. She graduated from Zhengzhou University in 2020, majoring in computer science and technology. From 2019 to now, she is currently engaged in professional work in communication.

\section{Author Contributions:}

Bin Wu conducted research conception, design and data analysis.

Bingxin Yao wrote and tested the core algorithm and simulation.

Yin Yang conducted data collation and comparison.

Chaoran Zhou conducted data collection and manuscript preparation.

Ning Zhu conducted data collection and discussion.

Creative Commons Attribution License 4.0 (Attribution 4.0 International, CC BY 4.0)

This article is published under the terms of the Creative Commons Attribution License 4.0

https://creativecommons.org/licenses/by/4.0/deed.en_US 